PROCEEDINGS OF THE

AMERICAN MATHEMATICAL SOCIETY

Volume 133, Number 4, Pages 975-977

S 0002-9939(04)07651-8

Article electronically published on August 4, 2004

\title{
ON A THEOREM OF AX
}

\author{
SHULIM KALIMAN
}

(Communicated by Michael Stillman)

\begin{abstract}
We show that in the case of affine and complete algebraic varieties over an algebraically closed field of zero characteristics any endomorphism of such a variety, that is injective on the complement to a subvariety of codimension 2 , is an automorphism.
\end{abstract}

In $1969 \mathrm{~J} . \mathrm{Ax}$ A proved a remarkable theorem which implies that every injective endomorphism of an algebraic variety $X$ (over an algebraically closed field $k$ of characteristic 0) is surjective. A. Borel [B] gave another proof of this result based on a cohomology-theoretic argument. A similar approach was used by Kawamata who rediscovered this theorem later $[\mathrm{I}]$. M. Miyanishi $[\mathrm{OP}]$ asked whether the following generalization of this result is true.

Conjecture. Let $\varphi: X \rightarrow X$ be an endomorphism of an algebraic variety $X$ over $k$, and let $E$ be a closed subvariety of $X$ of codimension at least 2. Suppose that the restriction of $\varphi$ to $X \backslash E$ is injective. Then $\varphi$ is an automorphism.

Unlike the Ax theorem this conjecture cannot be extended to schemes.

Example. Let $X$ be the union of two samples of $\mathbf{C}^{n}$ glued along $\mathbf{C}^{n} \backslash o$ where $o$ is the origin, i.e. the preimage $E$ of $o$ under the natural projection $X \rightarrow \mathbf{C}^{n}$ consists of two points $x_{1}$ and $x_{2}$. Consider the endomorphism $\varphi: X \rightarrow X$ that is identical on $X \backslash E$ and sends $E$ to $x_{1}$. This is the desired counterexample.

Nevertheless, following the idea of Borel and Kawamata, we shall show that the answer is positive in the case of affine and complete algebraic varieties.

Lemma 1. Let $\varphi: X \rightarrow X$ be a bijective endomorphism of an algebraic variety over a field $k$ of characteristic zero. Then $\varphi$ is an automorphism.

Proof. Consider a normalization $\nu: Y \rightarrow X$ of $X$. Then $\varphi$ generates a bijective endomorphism $\psi: Y \rightarrow Y$. Since $k$ is of zero characteristic this means that $\psi$ is birational and, therefore, an automorphism by the Zariski Main theorem. Let $\mathcal{R}$ and $\mathcal{S}$ be the structure sheaves on $Y$ and $X$ respectively. Treat $\mathcal{S}$ as a subsheaf of $\mathcal{R}$ (by identifying $\mathcal{S}$ with $\nu^{*}(\mathcal{S})$ ). Put $\mathcal{S}_{1}=\left(\psi^{*}\right)^{-1}(\mathcal{S})$ and $\mathcal{S}_{i}=\left(\psi^{*}\right)^{-1}\left(\mathcal{S}_{i-1}\right)$. Assume that $\mathcal{S}_{1} \neq \mathcal{S}$. Then $\mathcal{S}_{i} \neq \mathcal{S}_{i-1}$ since $\psi^{*}$ is bijective, i.e. we get a sequence of strict inclusions $\mathcal{S} \subset \mathcal{S}_{1} \subset \mathcal{S}_{2} \subset \ldots$ On the other hand choose a finite affine open covering $\left\{X_{j}\right\}$ of $X$. Then $\left\{Y_{j}\right\}$ is an open affine covering of $Y$ where $Y_{j}=\nu^{-1}\left(X_{i}\right)$. The restriction $\left.\mathcal{R}\right|_{Y_{j}}$ (resp. $\left.\mathcal{S}\right|_{Y_{j}},\left.\mathcal{S}_{i}\right|_{Y_{j}}$ ) is generated by those of its global sections

Received by the editors June 5, 2002 and, in revised form, November 20, 2003.

2000 Mathematics Subject Classification. Primary 14E25, 14R10.

The author was partially supported by NSA grant MDA904-03-1-0009.

(C)2004 American Mathematical Society 
that form an affine domain $R^{j}$ (resp. $S^{j}, S_{i}^{j}$ ). Note that $R^{j}$ is a finitely generated module over $S^{j}$ and each $S_{i}^{j}$ is an $S^{j}$-submodule of $R^{j}$. Hence the ascending chain condition implies that the sequence $\left.\left.\left.\mathcal{S}\right|_{Y_{j}} \subset \mathcal{S}_{1}\right|_{Y_{j}} \subset \mathcal{S}_{2}\right|_{Y_{j}} \subset \ldots$ must be stationary. Since our covering is finite, the sequence $\mathcal{S} \subset \mathcal{S}_{1} \subset \mathcal{S}_{2} \subset \ldots$ must also be stationary, which is a contradiction. Thus $\mathcal{S}_{1}=\mathcal{S}$. Since $\varphi^{*}=\left.\psi^{*}\right|_{\mathcal{S}}$ we see that $\varphi^{*}$ is an automorphism.

Lemma 2. The Conjecture is true provided it is true for normal algebraic varieties.

Proof. Consider a normalization $\nu: Y \rightarrow X$ of $X$. Then $\varphi: X \rightarrow X$ generates an endomorphism $\psi: Y \rightarrow Y$. By the hypothesis $\psi$ is bijective. Let $\ell(x)$ be the number of points of $\nu^{-1}(x)$ for $x \in X, n=\max _{x \in X} \ell(x)$, and $X_{n}$ be the subvariety of $X$ that consists of all points $x$ such that $\ell(x)=n$. Since $\psi\left(\nu^{-1}(x)\right) \subset \nu^{-1}(\varphi(x))$ for every $x \in X$ and $\psi$ is bijective, $\ell(\varphi(x)) \geq \ell(x)$. Hence $\varphi(x) \in X_{n}$ for every $x \in X_{n}$, and by the same reason there is no $x_{1} \neq x$ such that $\varphi\left(x_{1}\right)=\varphi(x)$. That is, $\left.\varphi\right|_{X_{n}}: X_{n} \rightarrow X_{n}$ is injective and, therefore, surjective by the Ax theorem. Thus $\varphi\left(X \backslash X_{n}\right) \subset X \backslash X_{n}$. Replacing $X$ by $X \backslash X_{n}$ we see that $\varphi\left(X_{n-1}\right) \subset X_{n-1}$ and $\left.\varphi\right|_{X_{n-1}}: X_{n-1} \rightarrow X_{n-1}$ is injective. Induction implies that $\varphi: X \rightarrow X$ is injective; hence it is an automorphism by the Ax theorem and Lemma 1.

Lemma 3. Let $E$ be a closed subvariety of $X$ of codimension at least 2, and $\varphi$ : $X \backslash E \rightarrow X$ be an injective morphism. Let $F=X \backslash \varphi(X \backslash E)$. Then $\operatorname{dim} E=\operatorname{dim} F$.

Proof. Assume the contrary. Let, say, $m=\operatorname{dim} E<\operatorname{dim} F=n$ (the case of $\operatorname{dim} E>\operatorname{dim} F$ is similar). Without loss of generality we can suppose that $X$ is normal. Then by the Zariski Main theorem, $\varphi$ is an embedding. Hence $\varphi(X \backslash E)$ is open in $X$ and $F$ is closed. Let $F_{1}=F \cup \varphi(F \backslash E)$. Note that $F_{1}$ is closed and it contains more irreducible components of dimension $n$ than $F$ does. Let $E_{1}=E \cup \varphi^{-1}(E)$. Note that $E_{1}$ is closed, $\operatorname{dim} E_{1}=m$, and $\varphi^{2}$ generates an isomorphism between $X \backslash E_{1}$ and $X \backslash F_{1}$. Replacing $E$ (resp. $F, \varphi$ ) by $E_{1}$ (resp. $F_{1}, \varphi^{2}$ ) we increase the number of irreducible components of $F$ of dimension $n$; hence we can make it as large as we wish while keeping the dimension of $E$ the same.

Following the proof of Kawamata we shall use De Rham homology from $[\mathrm{H}$. For every $l$-dimensional variety $Y$ we have $H_{i}(Y)=0$ for $i>2 l$, and $H_{i}(Y)$ is a finite-dimensional $k$-vector space that is nonzero for $i=2 l$. Hence the MayerVietoris sequence implies that the dimension of $H_{2 l}(Y)$ is at least the number of irreducible $l$-dimensional components of $Y$. Furthermore, we have the following exact sequences [H, Th. 1.2]:

$$
\begin{aligned}
& \ldots \rightarrow H_{i}(E) \rightarrow H_{i}(X) \rightarrow H_{i}(X \backslash E) \rightarrow H_{i-1}(E) \rightarrow \ldots, \\
& \ldots \rightarrow H_{i}(F) \rightarrow H_{i}(X) \rightarrow H_{i}(X \backslash F) \rightarrow H_{i-1}(F) \rightarrow \ldots
\end{aligned}
$$

Since $m<n<j=\operatorname{dim} X$ we get from the first sequence $H_{i}(X) \simeq H_{i}(X \backslash E)$ for $2 j \geq i \geq 2 m+2$. Since $X \backslash E$ is isomorphic to $X \backslash F$ we have $H_{i}(X) \simeq H_{i}(X \backslash F)$ for $2 j \geq i \geq 2 m+2$. Hence from the second sequence we have

$$
0 \rightarrow H_{2 n+1}(X) \rightarrow H_{2 n+1}(X) \rightarrow H_{2 n}(F) \rightarrow H_{2 n}(X) \rightarrow \ldots .
$$

That is, we have a monomorphism $H_{2 n}(F) \rightarrow H_{2 n}(X)$. As we mentioned the dimension of the last $k$-vector space is bounded while we can make the dimension of $H_{2 n}(F)$ as large as we wish by increasing the number of components of $F$. This is the desired contradiction. 
Theorem. The Miyanishi Conjecture is true for affine and complete varieties.

Proof. Let us suppose first that $X$ is affine. By Lemma 2 we can also suppose that $X$ is normal. Note that $F$ does not contain irreducible components of codimension at least 2 since $\varphi^{-1}$ can be extended to these components by the theorem about deleting singularities of regular functions on normal varieties. Lemma 3 implies that $F$ and $E$ must be empty.

When $X$ is complete, then $F=\varphi(E)$ since $\varphi(X)=X$. Since we can assume normality, $\operatorname{dim} F<\operatorname{dim} E$ by the Zariski Main theorem whence $F$ and $E$ are empty by Lemma 3. Now Lemma 1 implies the desired conclusion.

Remark. The lemmas above remain true for an integral scheme $X$ of finite type over $k$ (in the case of algebraic varieties Lemma 3 can also be extracted from the "conservation property" proven in [G] by a metamathematical argument). The reason why the Miyanishi conjecture is not valid for such (non-separated) schemes is that the image $\varphi(X)$ may not be closed in $X$ as in the counterexample in the beginning of the paper.

\section{REFERENCES}

[A] J. Ax, Injective endomorphisms of varieties and schemes, Pacific J. Math., 31 (1969), 1-7. MR 0251036 (40:4267)

[B] A. Borel, Injective endomorphisms of algebraic varieties, Arch. Math. 20 (1969), 531-537. MR 0255545 (41:206)

[G] M. Gromov, Endomorphisms of symbolic algebraic varieties, J. Eur. Math. Soc. 1:2 (1999), 109-197. MR 1694588 (2000f:14003)

[H] R. Hartshorne, Algebraic De Rham cohomology, Manuscripta Math. 7(1972), 125-140. MR $0313255(47: 1810)$

[I] S. Iitaka On logarithmic Kodaira dimension of algebraic varieties, In: Complex Analysis and Algebraic Geometry, 175-189, Kinokuniya, Tokyo, 1977. MR 0569688 (58:27975)

[OP] Open Problems, Workshop on Group Actions on Rational Varieties, edited by G. Freudenburg and P. Russell, CICMA Report 2002-04 (to appear).

Department of Mathematics, University of Miami, Coral Gables, Florida 33124

E-mail address: kaliman@math.miami.edu 